\title{
A PERCEPÇÃO DE PORTADORES DE INSUFICIÊNCIA CARDÍACA SOBRE SEU SUPORTE SOCIAL
}

Verônica Schneider Röder

Universidade Luterana do Brasil

Aline Groff Vivian

Universidade Luterana do Brasil
Recebido em: 07/05/2020

$1^{\text {a }}$ revisão em: 19/05/2021

Aceito em: 04/09/2021

\section{RESUMO}

A Insuficiência Cardíaca (IC) é definida como uma incapacidade do coração de manter o bombeamento cardíaco adequado. A doença traz consequências como mudanças na rotina para adaptação, o que pode gerar isolamento social nos portadores. O artigo descreveu o suporte social percebido pelos pacientes com IC. Trata-se de um estudo qualitativo exploratório-descritivo, com 12 participantes com idades entre 65 e 80 anos, integrantes de Programa de Reabilitação Cardiorrespiratória, atendidos por equipe multidisciplinar. Foram aplicadas a Escala de Satisfação com Suporte Social (ESSS) e uma entrevista semiestruturada. Os participantes apresentaram resultados variados de 75, próximo do máximo até 29 pontos na ESSS. Quanto às percepções, relataram receber apoio emocional pela maior parte da família e apresentaram cuidado em termos de saúde física. Alguns mencionaram dificuldade nas relações sociais devido à IC. A partir dessa pesquisa, intervenções específicas para esse público podem ser melhor delineadas, assim como novos estudos com população maior.

Palavras-chave: suporte social; pacientes cardíacos; intervenção em grupo. 


\section{HEART FAILURE PATIENTS' PERCEPTIONS ABOUT THEIR SOCIAL SUPPORT}

\section{ABSTRACT}

Heart Failure (HF) is defined as an inability of the heart to maintain adequate cardiac output. The disease leads patients to consequences such as changes in adaptation routine, which might lead to social isolation. The aim of the study is to describe the social support perceived by patients with HF. This is a qualitative exploratory and descriptive study, with 12 participants aged between 65 and 80 years old, member of a Cardiorespiratory Rehabilitation Program, assisted by a multidisciplinary team. The Social Support Satisfaction Scale (ESSS) and a semistructured interview were applied. Participants had scores ranging from 75, close do the maximum to 29 points on the ESSS. The patients' perceptions reported emotional support received by their families, and they showed care regarding their physical health. Some mentioned difficulties in social relations due to HF. Considering this research, specific interventions for this audience can be better designed, as well as other studies with a larger population.

Keywords: social support; cardiac patients; group intervention.

\section{LA PERCEPCIÓN DE LOS PACIENTES CON INSUFICIENCIA CARDÍACA SOBRE SU APOYO SOCIAL}

\section{RESUMEN}

La insuficiencia cardíaca (IC) se define como la incapacidad del corazón para mantener un bombeo cardíaco adecuado. La enfermedad trae consecuencias como cambios en la rutina, lo que puede generar aislamiento social. El objetivo del estudio fue describir el apoyo social percibido por los pacientes portadores de la IC. Es un estudio cualitativo, exploratorio descriptivo, en el que participaron 12 participantes de entre 65 y 80 años, integrantes del Programa de Rehabilitación Cardiorrespiratoria, atendidos por un equipo multidisciplinario. Se aplicó la Escala de Evaluación de Apoyo Social (ESSS) y una entrevista semiestructurada. Los participantes tuvieron resultados variados desde 75, cerca del máximo a 29 puntos en la ESSS. En cuanto a las percepciones ellos reportaron el apoyo emocional en gran parte por la familia y presentando atención en términos de salud física. Algunos mencionaron dificultades en las relaciones sociales debido a la IC. Con base em esta investigación, se pueden diseñar mejores intervenciones específicas para esta audiencia, así como nuevos estudios con una población más grande.

Palabras clave: apoyo social; pacientes cardíacos; intervención grupal. 


\section{INTRODUÇÃO}

A insuficiência cardíaca (IC) é definida por Engel e colaboradores (2011) como "uma condição em que o coração não consegue bombear o sangue de acordo com a demanda tecidual, ou só o faz à custa de aumentos na pressão de enchimento" ( $p$. 11). A preocupação dispensada a tal condição se deve ao fato de que as doenças cardiovasculares são, no Brasil, as principais causadoras de óbitos. O crescimento do número de pessoas com IC está relacionado ao envelhecimento da população mundial proveniente do aumento da expectativa de vida (Ishigaki, Hanna, Castro, Holanda \& Castro, 2021). A condição é considerada uma epidemia, gerando um quadro de alta mortalidade e morbidade (Bocchi et al, 2012). Em nosso país, a taxa de mortalidade é de 14 para cada 100.000 casos (Fernandes et al, 2020).

Segundo o DATASUS, a região brasileira com maior número de óbitos por cardiopatias é a Sudeste, seguida pelo Nordeste, sendo esses dados relativos ao ano de 2019 (Ministério da Saúde, 2019). Em relação ao perfil das pessoas portadoras de insuficiência cardíaca no Brasil, sabe-se que a média de idade se encontra em 64 anos e o sexo predominante é o feminino, representando $60 \%$ dos casos. Já a etnia branca alcançou a porcentagem de $59 \%$, sendo a mais atingida. A insuficiência cardíaca apareceu atrelada à hipertensão em boa parte dos casos. Comorbidades emocionais, como a depressão, também foram apontadas como frequentes em pacientes cardíacos (Bocchi et al, 2012; Tinoco et al, 2021). Além disso, a partir do início do tratamento, os cardiopatas precisam lidar com outras complicações, como labilidade emocional, aumento da sensibilidade, e até micções mais frequentes, o que, em alguns casos, pode gerar maior isolamento social (Queiroz \& Foz, 2018).

Sendo a Insuficiência Cardíaca considerada uma síndrome crônica, que afeta de modo individual a cada pessoa acometida, é importante que se considerem fatores biopsicossociais dos indivíduos no tratamento da mesma (Barbosa, Franklin, Stefenoni, Moraes, Jacques, Serpa, Calil, \& Barbosa, 2014). O paciente portador da Insuficiência Cardíaca costuma sentir medo da morte, visto que o coração é um órgão representante da vida, o que o afeta emocionalmente. Tais sintomas psíquicos aumentam em casos graves, quando há necessidade de transplante. (Mendes \& Eufrásio, 2013). A dor, relacionada ao quadro clínico, também tem repercussões emocionais, podendo afetar inclusive o sistema nervoso por meio da liberação de substâncias (Soares, Toledo, Santos, Lima \& Galdeano, 2008). Pacientes idosos costumam demandar uma rede de apoio multiprofissional e familiar, especialmente decorrente das limitações diante do envelhecimento e da crescente dependência que pode surgir com os sintomas do adoecimento pela IC (Oliveira, Souto \& Vitório, 2017). A educação em saúde, ao viabilizar conhecimentos e favorecer a adesão ao tratamento também é destacada como forma de promoção da saúde (Queiroz et al, 2021). 


\section{SUPORTE SOCIAL}

O suporte social pode ser definido de diversas formas, considerando-se fatores quantitativos e qualitativos. O conceito também se define em relação às estruturas das relações sociais. A forma como o suporte social é mais frequentemente avaliado diz respeito aos aspectos: afetivo-emocional, instrumental, ajuda ou informação. Como atividades de suporte, pode-se exemplificar: expressar preocupação, demonstrar afeto, partilhar uma atividade, prestar cuidados, aconselhar, dar sugestões e socializar (Ornelas, 1994). Sendo assim, esse suporte envolve apoio emocional, informação ou orientação para resolução de problemas, apoio efetivo, carinho e companheirismo. Na população idosa, torna-se fundamental, em especial, diante da vulnerabilidade decorrente do aumento de doecimento (Hwang, Fleischmannn, Howie-Esquivel, Stotts \& Dracup, 2011; Cestari, Florêncio, Pessoa \& Moreira, 2021).

\section{SUPORTE SOCIAL E INSUFICIÊNCIA CARDÍACA}

Além da atenção necessária para questões emocionais, o olhar para o suporte social mostra-se importante em pacientes com insuficiência cardíaca porque, segundo estudos, as redes de apoio favorecem a adesão ao tratamento, obtendose melhores resultados no tratamento das doenças do coração do que em pacientes que obtiveram tratamento médico exclusivamente (Rodrigues \& Sildl, 2008; Molano-Barrera \& González-Consuegra, 2021). Ademais, estudo com pacientes cardíacos constataram alto nível de apoio emocional entre os mesmos, sendo esta uma das possíveis razões, devido ao fato de o coração ser considerado um órgão nobre, o que pode causar mobilização dos familiares em favor dos pacientes. Notou-se uma relação significativa quanto ao nível de apoio e os níveis de escolaridade, sendo que quanto maior a escolaridade, menor o grau de apoio social percebido. Esse fator esteve relacionado ao aumento da capacidade crítica de acordo com os níveis de escolaridade. Além disso, o estudo considerou importante levar em conta o fato de a cultura considerar negativo avaliar como ineficiente a rede de apoio, o que pode ter influenciado as respostas dos participantes (Dantas, Pelegrino \& Garbin, 2007).

Outros estudos demonstraram que, mesmo com suporte social pouco expressivo, grande parte dos pacientes cardiopatas consideram-se satisfeitos com o mesmo. Dentre as pessoas que mais prestaram apoio social para esses pacientes estavam amigos e familiares. Não foram citados colegas, nem mesmo profissionais de saúde na maior parte dos casos. É importante mencionar que, apesar de os pacientes apresentarem-se satisfeitos com o suporte social mínimo, tal carência de relações pode ocasionar até mesmo danos à saúde física, como aumento da pressão arterial (Rezende, Mendes \& Santos, 2007; Cestari et al, 2021).

No processo de mudança desencadeado pela insuficiência cardíaca, muitos pacientes consideram como fator facilitador na adaptação, o apoio da família. É importante pontuar que o impacto trazido pela doença impede que os pacientes realizem atividades outrora prazerosas a estes, o que afeta cotidianamente o 
convívio social. As dificuldades como as de locomoção influenciam no status social do paciente, que por vezes aumento o isolamento. Nesses casos, a família pode ser fator protetor na adaptação (Mendes, Bastos \& Paiva, 2010).

Há ainda a fadiga relacionada ao quadro clínico, que dificulta a realização de algumas atividades, repercutindo nas relações sociais dos pacientes com insuficiência cardíaca. Já com a manutenção de uma rede de suporte social o paciente pode ter aumento da autoestima, entre outros benefícios (Santos, Santo, Pestana, Daher \& Santana, 2011). Também surge a incerteza quanto à manutenção da vida laboral, que afeta boa parte dos pacientes. Alguns passam a apresentar isolamento social e humor deprimido por não conseguirem manter o mesmo desempenho ocupacional (Teixeira, Masuchi \& Correia, 2017). Durante internações hospitalares é comum que a saúde mental seja afetada ocasionando até mesmo o surgimento de depressão, sendo que os que desenvolvem tal transtorno geralmente possuem menor apoio e maior isolamento social (Marques, Stefanello, de Mendonça, \& Furlanetto, 2013).

Por não conseguirem desempenhar algumas atividades mais complexas que envolvem papéis sociais importantes, muitos idosos acabam apresentando baixa frequência de envolvimento social. Tal dado mostra-se preocupante tendo em vista o papel protetor para a saúde mental do indivíduo que o suporte social proporciona. Ademais, há uma relação entre sintomas de ansiedade elevados e baixo suporte social. Já quando há a percepção de que existe alguém disponível para suporte emocional material ou instrumental, as tensões podem tornar-se menores (Possatto \& Rabelo, 2017). Em estudo feito com cuidadores de pacientes com Insuficiência Cardíaca, aqueles que exerciam cuidado relataram uma repercussão positiva de suas ações, visto que os pacientes tiveram melhora clínica e psicológica, passando inclusive a se cuidar melhor. Além disso, os cuidadores ressaltaram a importância de o paciente saber que alguém se preocupa com ele (Campos, Martins \& Carvalho, 2019). Outro impacto do suporte social, diz respeito à adesão ao tratamento medicamentoso. Uma pesquisa demonstrou que o número de amigos próximos e parentes vistos no último mês se mostrou moderadamente associado com maior adesão medicamentosa. Um suporte social funcional e estruturado mostrou-se então de grande importância (Mondesir et al, 2018).

O suporte social, diante do exposto, pode incluir vários fatores. Dentre eles: suporte emocional, feedback, aconselhamento ou orientação, assistência prática, financeira e socialização (Ornellas, 1994; Hwang et al, 2011; Oliveira, Souto \& Vitório, 2017). Tendo em vista o que foi apresentado sobre benefícios do suporte social efetivo e prejuízos quando o mesmo não é funcional, nota-se a importância de abordar o tema com pacientes idosos que apresentam insuficiência cardíaca. Sendo assim, o presente trabalho visa descrever o suporte social percebido por pacientes portadores de insuficiência cardíaca, levando em consideração os aspectos do apoio social. 


\section{MÉTODO}

Trata-se de um estudo qualitativo, exploratório-descritivo. Participaram 12 pacientes, com diagnóstico de Insuficiência Cardíaca (IC), com idades entre 65 e 80 anos, sendo quatro homens e oito mulheres, residentes na região metropolitana de Porto Alegre/RS. A amostra foi escolhida por acessibilidade, perante a participação de idosos em grupos de intervenção psicológica, do Ambulatório de Insuficiência Cardíaca, do Programa de Reabilitação Cardiorrespiratória, de uma universidade privada do sul do Brasil. Todos os participantes integravam o projeto referido, que prestava assistência aos pacientes, por equipe multidisciplinar, composta de professores e acadêmicos de Medicina, Fisioterapia, Educação Física, Biomedicina e Psicologia. A coleta de dados foi realizada pela primeira autora deste estudo, sob orientação da segunda pesquisadora e ocorreu entre março e julho de 2019, totalizando 4 encontros. A descrição pormenorizada da estrutura dos grupos conduzidos por profissionais da Psicologia, teve seu funcionamento detalhado descrito por Finkler e Vivian (2018).

Os pacientes que aceitaram participar da pesquisa assinaram o Termo de Consentimento Livre e Esclarecido (TCLE). Preencheram também a ficha de dados sociodemográficos. Foi aplicada ainda, a Escala de Satisfação com Suporte Social (ESSS), que possui 15 itens, com 5 opções em escala likert: "concordo totalmente", "concordo na maior parte", "não concordo nem discordo", "discordo na maior parte" e "discordo totalmente". A ESSS divide-se em quatro fatores: "satisfação com os amigos", "intimidade", "satisfação com a família" e "atividades sociais" (Ribeiro, 1999). Por fim, foi aplicada uma entrevista semiestruturada sobre a satisfação com suporte social, com questões que diziam respeito à percepção dos indivíduos sobre seu suporte social, tais como: Como você descreveria as suas relações sociais? Você se sente apoiado por amigos e familiares? Por quê? Com que frequência você visita ou é visitado por amigos ou familiares? Como é a qualidade do tempo que você passa com amigos ou familiares? Entre outras.

As entrevistas foram gravadas e transcritas. A sistematização dos dados em categorias foi realizada a partir da Análise de Conteúdo qualitativa proposta por Bardin (2016), que consiste em três etapas: pré-análise, exploração do material e tratamento dos resultados, inferência e interpretação. A partir daí, os relatos foram organizados em categorias temáticas derivadas da literatura e da fala das participantes. Dois dos autores do presente estudo classificaram separadamente os trechos relativos às percepções e sentimentos acerca do suporte social recebido pelos idosos, em relação ao suporte afetivo-emocional, cuidado em termos de saúde física, expressão de preocupação e relações sociais.

Os procedimentos de coleta foram realizados respeitando os procedimentos éticos, diretrizes e normas estabelecidas na Resolução n. 466/12 do Conselho Nacional de Saúde - CNS, que regulamenta as pesquisas envolvendo seres humanos. A pesquisa foi aprovada pelo Comitê de Ética em Pesquisa da Universidade Luterana do Brasil, sob o CAAE: 53116716.4.0000.5349, de 2016. 


\section{RESULTADOS}

O público alvo do estudo foi composto por 12 pacientes, diagnosticados com Insuficiência Cardíaca, sendo 8 mulheres e 4 homens, com idades entre 65 e 80 anos, residentes da região metropolitana de Porto Alegre, com escolaridade e estado civil variados e apenas um participante com atividade laboral ativa.

\section{Dados sociodemográficos dos participantes}

\begin{tabular}{|c|c|c|c|c|c|}
\hline Nome & Sexo & Idade & $\begin{array}{l}\text { Estado } \\
\text { Civil }\end{array}$ & Escolaridade & Profissão \\
\hline E. S. R. & Feminino & 65 & Viúva & $\begin{array}{l}\text { Ensino } \\
\text { Médio }\end{array}$ & Aposentada \\
\hline M. B. & Feminino & 69 & Divorciada & $\begin{array}{l}\text { Ensino } \\
\text { Fundamental }\end{array}$ & Aposentada \\
\hline N. A. P. & Feminino & 72 & Casada & $\begin{array}{l}\text { Ensino } \\
\text { Fundamental }\end{array}$ & Aposentada \\
\hline F. A. M. & Masculino & 66 & Divorciado & $\begin{array}{l}\text { Ensino } \\
\text { Médio }\end{array}$ & $\begin{array}{c}\text { Representante } \\
\text { comercial }\end{array}$ \\
\hline A. M. M. S. & Feminino & 69 & Casada & $\begin{array}{l}\text { Ensino } \\
\text { Fundamental }\end{array}$ & Do lar \\
\hline M. L. B. S. & Feminino & 76 & Casada & $\begin{array}{l}\text { Ensino } \\
\text { Médio }\end{array}$ & Aposentada \\
\hline L. S. P. & Feminino & 80 & Viúva & $\begin{array}{l}\text { Ensino } \\
\text { Fundamental }\end{array}$ & Do lar \\
\hline W. A. M. & Feminino & 71 & Viúva & $\begin{array}{l}\text { Ensino } \\
\text { Fundamental }\end{array}$ & Aposentada \\
\hline A. F.S. & Masculino & 74 & Casado & $\begin{array}{l}\text { Ensino } \\
\text { Fundamental }\end{array}$ & Aposentado \\
\hline F. B. & Masculino & 67 & Casado & $\begin{array}{l}\text { Ensino } \\
\text { Fundamental }\end{array}$ & Aposentado \\
\hline O. C. & Masculino & 65 & Casado & $\begin{array}{l}\text { Ensino } \\
\text { Médio }\end{array}$ & Aposentado \\
\hline Z.P.I. & Feminino & 72 & Casada & $\begin{array}{l}\text { Ensino } \\
\text { Médio }\end{array}$ & Do lar \\
\hline
\end{tabular}

A seguir, a Figura 1 apresenta os dados referentes à Escala de Satisfação com Suport Social. A ESSS tem uma variação de 15 (pontuação mínima) a 75 (pontuação 
máxima), sendo que quanto maior o resultado alcançado, maior a satisfação com o suporte social.

Pode-se notar pela Figura 1, que 5 pacientes apresentaram satisfação significativa com seu suporte social, chegando próximos à pontuação máxima do instrumento, embora ninguém a tenha alcançado. Os demais pacientes tiveram uma variação entre 49 e 29 pontos, sendo esse último valor atingido por apenas um paciente, seguida de 39 pontos (pontuação de dois pacientes). A média e a mediana encontradas foram respectivamente 53,25 e 48 pontos.

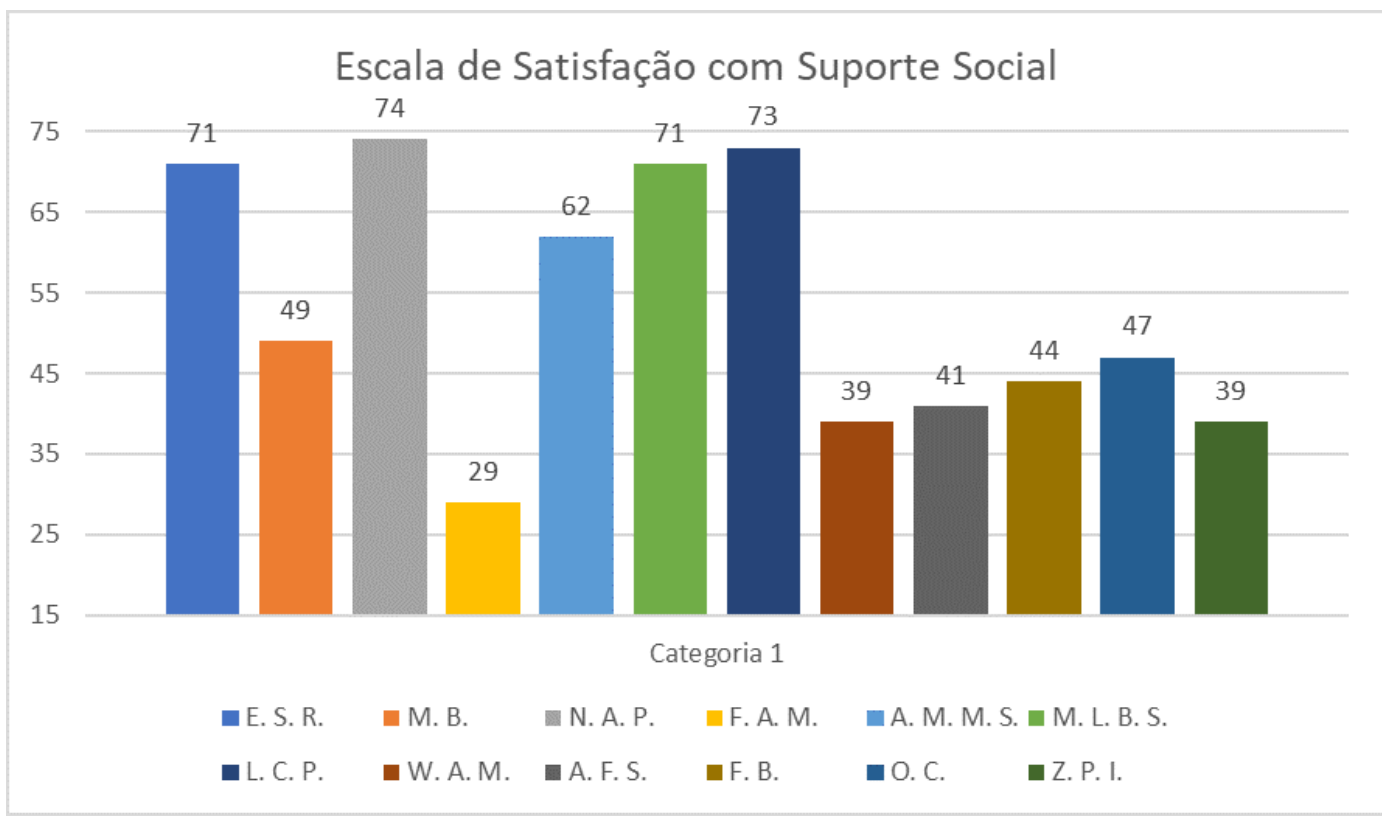

Figura 1. Dados da Escala de Satisfação com Suporte Social

A seguir serão descritos os resultados da análise qualitativa de conteúdo, a partir das falas dos pacientes, obtidas nas entrevistas e analisadas em categorias e subcategorias.

\section{PERCEPÇÕES E SENTIMENTOS ACERCA DO SUPORTE SOCIAL RECEBIDO PELOS PACIENTES}

As percepções apresentadas pelos pacientes foram divididas em categorias, de acordo com as áreas de suporte social, mencionadas por Ornelas (1994): suporte afetivo-emocional, cuidado em termos de saúde física, expressão de preocupação e relações sociais.

\section{SUPORTE AFETIVO-EMOCIONAL}

Nessa primeira subcategoria, os pacientes trouxeram à tona o suporte afetivoemocional recebido de amigos ou familiares. Dentre eles, alguns relataram um suporte efetivo, conforme as falas abaixo: 
"Ah, eu não sei te dizer, é que tudo que eu tiver que conversar com eles, eles me acolhem, tanto família quanto amigos" (N, 72 anos); "Eles às vezes me vêem e eles me conhecem. Eles sabem que eu sou muito brincalhona, eu sou assim. E se ele vêem que eu tô quieta, e: 'o que aconteceu?' 'a senhora se incomodou?' 'a senhora tá bem?'" (M, 76 anos).

Outros relatos abordaram a importância da família quanto ao acolhimento afetivoemocional, conforme as falas a seguir:

"Geralmente só com a família... Desabafa alguma coisa que a gente sente, amigo não... Geralmente só os mais próximos, mulher e filhos." (A, 74 anos); "Ah, não tenho amigo íntimo, a única é minha nora... que qualquer coisa que eu tenho eu desabafo com ela." ( $L, 80$ anos).

Houve também alguns participantes que consideraram não possuir suporte afetivo-emocional, sem ter alguém para desabafar quando necessário:

"Aí é raro, eu não desabafo com ninguém, eu trago mais mesmo para o meu controle. Comigo mesma, é." (W, 71 anos); "Amigo íntimo? Eu não tenho amigo íntimo. E para desabafar eu não tenho ninguém." ( $F, 66$ anos).

\section{CUIDADO EM TERMOS DE SAÚDE FÍSICA}

Em relação ao cuidado com a saúde física, todos expressaram poder contar com os familiares, manifestando satisfação com o apoio recebido nesse âmbito:

"Eu me sinto... satisfeita com o apoio que eu tenho. Porque, vamos dizer assim, de lidar com esses problemas de doença eles estão sempre presentes comigo." (W, 71 anos); "Ah, eu me sinto muito apoiada. Em tudo. Porque se eu precisar pra saúde, qualquer coisa que eu precisar, eu tenho, posso contar." (A, 69 anos). E também puderam pedir ajuda: "Médio. Porque, não sei, eles... Acho que nunca pedi nada para eles também. Médio, talvez porque quando eu machuquei o braço, daí minha irmã foi pra me ajudar. As pessoas de perto vêm visitar." (M, 69 anos).

Apesar de poderem contar com seus familiares em momentos que apresentam problemas de saúde, um dos pacientes referiu o fato de as pessoas só ajudarem em situações relacionadas a emergências: 
"Porque a gente é assim, sabe? Eu vivo a minha vida, eles vivem a vida deles. Se precisar, ah, caso de emergência aí eu acho que tudo bem, a gente cada um ajuda o outro. Mas enquanto não houver essa emergência, então cada um vive no seu canto." ( $F$, 66 anos).

\section{EXPRESSÃO DE PREOCUPAÇÃO}

A maioria dos pacientes sentiu-se satisfeito com a preocupação demonstrada por amigos e familiares, através de orientações e ajuda:

"Bem, bem apoiado. Porque eu sou muito paparicado, eles não me deixam fazer nada. Tudo: 'tu fica quieto, que nós fazemos'."' (O, 65 anos); "Ai, o máximo. Eu vou botar 99\%. Porque tudo que eles fazem é pra me ajudar, sabe. Tudo que eu preciso... semelhante a essas coisas que o ser humano necessita." ( $Z, 72$ anos); "É, isso aí eu me sinto bem." (F, 67 anos). Apenas um dos pacientes relatou receber apoio suficiente: "Apoiado? Por amigos? Pra começar, eu não tenho amigos, então não tenho apoio. E familiares, também não. Aliás, às vezes eu ajudo eles." ( $F$, 66 anos).

\section{RELAÇÕES SOCIAIS}

A categoria de relações sociais diz respeito às interações com amigos, familiares ou outras pessoas. Nesse sentido, os participantes relataram visitar ou ser visitados por amigos e familiares:

"É, amigos eu que visito mais. Agora os familiares me visitam toda a semana. Se eu não vou na casa deles, eles vêm na minha casa. Então é toda a semana. É aquele ritual. Eu já sei, todo o domingo eles vêm pra minha casa, né." ( $E, 65$ anos).

Outros expressaram a capacidade de manter boas relações, referindo não enfrentar problemas no relacionamento social:

"Acho que eu sou uma pessoa que procuro sempre me relacionar com o que tiver de oportunidades, eu tô sempre pronta para participar." ( $Z, 72$ anos); "Eu acho que é uma relação social mais com a família, com os amigos, meus vizinhos, me dou bem com todo mundo, tô sempre tranquila. Não tenho problema com ninguém." ( $L, 80$ anos). 
Já outro participante mencionou ter dificuldade em manter interações sociais, relacionando a isso, seus problemas de saúde, como a Insuficiência Cardíaca:

"Ah, sociais. Não está bem porque não tô conseguindo a vida social. Eu sinto cansaço, sabe? Então eu não tenho a vontade de sair, passear e aquele cansaço. Da insuficiência, que eu tenho também é, sou diabético. O diabético já dá um cansaço, mais a insuficiência. Então, eu tô me recuperando aqui. Só que eu tô melhorando, né." (F, 66 anos).

Não foram encontrados relatos dos pacientes em relação à assistência prática: financeira ou material, o que pode ser explicado pelo fato de muitos deles ainda manterem certa independência financeira, muitas vezes residindo sozinhos.

\section{DISCUSSÃO}

Tendo em vista os resultados apresentados a partir das falas dos pacientes entrevistado, e com base na literatura que expressa as várias formas de suporte social (Oliveira, Souto \& Vitório, 2017; Ornellas, 1994), tornou-se possível compreender a percepção dos pacientes portadores da Insuficiência Cardíaca (IC) sobre seu suporte social, em termos de suporte afetivo-emocional, cuidado em termo de saúde física, expressão de preocupação e relações sociais, que serão discutidas a seguir.

Inicialmente, foram descritas as características sociodemográficas do grupo, como sexo, idade, nível educacional, estado civil e profissão. O sexo feminino mostrouse o mais presente na pesquisa, e média de idade foi 70 anos, o que corrobora a literatura que demonstra maior prevalência de mulheres, perfazendo $60 \%$ dos casos com a doença no Brasil. Já a média de idade mostrou-se maior na presente pesquisa quando em comparação à literatura, que aponta essa faixa como 64 anos, para pacientes cardiopatas no Brasil (Bocchi et al, 2012).

Sobre a ocupação obteve-se maior incidência de aposentados no presente estudo. Não foram encontrados dados concretos da prevalência de determinadas ocupações em grupos de pacientes com IC na literatura, porém, Teixeira et al (2017) afirmam que a não manutenção de atividades laborais é frequente em tal grupo, devido às limitações da doença, o que levaria os mesmos a aposentarem-se. Em relação ao nível educacional e estado civil notou-se maior frequência de ensino fundamental incompleto e casados respectivamente, semelhante ao apontado por Silva et al (2021), que investigou a qualidade de vida e apontou que os portadores de IC eram em sua maioria casados, com ensino fundamental.

Neste estudo foi aplicada a Escala de Satisfação com Suporte Social (ESSS), que mede a satisfação a partir de cinco fatores relacionados ao suporte social "satisfação com os amigos", "intimidade", "satisfação com a família" e "atividades 
sociais", com escores que variam de 15 a 75 (Ribeiro, 1999). Nos resultados, observou-se que cinco participantes do presente estudo atingiram de 71 a 74 pontos, o que significa que esses participantes estão muito satisfeitos com sua relação com amigos, familiares, bem como relações sociais e íntimas. Outros seis pacientes apresentaram escore mediano, obtendo de 39 a 49 pontos, o que mostra que os mesmos estão satisfeitos em parte com o suporte social recebido. Houve ainda um paciente com escore 29, o que pode ser considerada uma pontuação baixa, representando insatisfação, em grande parte, com seu suporte social. Diferente desses achados, os participantes com IC, do estudo de Dantas et al (2007) apresentaram escores altos, ou seja, a maioria dos pacientes estavam satisfeitos com o suporte recebido. Os pesquisadores acreditam que as altas pontuações poderiam estar relacionadas ao fato de alguns familiares realmente prestam maior suporte aos pacientes, tendo em vista o coração ser considerado um órgão nobre ou, ainda, de que é socialmente inaceitável para muitas pessoas avaliarem a rede de apoio como insatisfatória. Os pacientes apresentaram com mais frequência, no presente estudo, índices medianos de satisfação com suporte social e não somente escores altos, conforme citado no parágrafo acima, o que mostra uma visão mais realista sobre o suporte social recebido, mesmo que os participantes apresentem, em sua maioria, nível educacional baixo. Tal dado diferencia-se do estudo feito por Dantas et al (2007), cuja avaliação de índices menores de suporte social se deu em pessoas com maior escolaridade.

No que diz respeito às percepções e sentimentos acerca do apoio social recebido pelos pacientes, foram considerados os relatos sobre o suporte afetivo-emocional, cuidado em termos de saúde física, expressão de preocupação e relações sociais. Em termos de suporte afetivo-emocional, alguns participantes destacaram um suporte efetivo. Outros relataram maior suporte por parte da família. E houve ainda os que consideraram não ter suporte afetivo-emocional. Em relação a tal aspecto, a literatura aponta que a presença de alguém que possa prestar suporte emocional facilita as demandas do dia a dia, podendo diminuir tensões e favorecer até mesmo o cuidado com a saúde física e adesão à medicação (Mondesir et al, 2018). A vulnerabilidade do portador de IC não está somente relacionada a questões físicas, pois inclui suas relações de cuidado afetivo, mas também ao envelhecimento que se encontra presente na maior parte dos pacientes acometidos de cardiopatias (Possatto \& Rabelo, 2017; Cestari, Florêncio, Pessoa \& Moreira, 2021). Outro estudo está de acordo com o fato de os familiares serem mais frequentes no suporte emocional, juntamente com amigos, não sendo citados colegas ou profissionais de saúde (Rezende et al, 2007).

O cuidado em termos de saúde física foi salientado como eficiente por todos os pacientes, os quais deixaram claro que puderam e poderiam contar com amigos e familiares em situações de emergência ou para cuidados básicos. Apesar disso, um dos pacientes considerou que realmente era necessário ocorrer uma emergência para que tal cuidado fosse observado, o que foi considerado insatisfatório. A literatura demonstra a importância do cuidado em momentos de fragilidade na saúde física, uma vez que a falta do mesmo pode gerar transtornos psíquicos como 
a depressão. Já a presença de suporte social nesse momento mostra-se fator protetor inclusive em termos de saúde mental (Marques et al, 2013; Cestari et al 2021).

No quesito expressão de preocupação os participantes relataram notar em seus familiares tal demonstração através de conselhos, cuidados, e preservação dos mesmos em atividades. Apesar disso, um paciente referiu não perceber preocupação por parte dos outros. O estudo trazido por Campos et al (2019) relata a mesma realidade dos participantes do estudo, em que os pacientes possuíam um cuidador que se preocupava com os mesmos e obtiveram inclusive melhora física. $\mathrm{Na}$ visão dos próprios cuidadores, apenas notar a preocupação de alguém com eles já pode ser de grande importância (Campos et al., 2019).

Quanto à categoria relações sociais, os pacientes verbalizaram visitar e ser visitados por familiares e amigos. Já outros, manifestaram ter a capacidade de manter boas relações, não apresentando problemas de relacionamento. Em relação à fala dos pacientes de que consideram suas relações sociais suficientes, encontra-se na literatura que muitos dos pacientes com insuficiência estão satisfeitos com suas relações, mesmo que as mesmas sejam pobres. É importante ressaltar que, mesmo os pacientes apresentando-se satisfeitos com o suporte social mínimo, uma pobreza de relações pode trazer danos à saúde, o que pode gerar inclusive aumento da pressão arterial. É também sabido que, os pacientes com suporte social adequado, apresentam resultados mais promissores no tratamento da IC e adesão medicamentosa (Rezende, et al, 2007; Mondesir et al., 2018; MolanoBarrera \& González-Consuegra, 2021).

Por fim, na mesma categoria que se referiu aos relacionamentos, outro participante trouxe à tona sua dificuldade em manter relações pessoais, devido às limitações ocasionadas pela insuficiência cardíaca. Tal dado está de acordo com Mendes et al (2010), que apontam para o impacto da doença, tais como dificuldades de locomoção, influenciando o status social, impedindo os pacientes de manterem as mesmas atividades de lazer, o que afeta seu convívio social. Esse aspecto foi corroborado por Santos et al. (2011), que apontam que a fadiga relacionada ao quadro clínico repercute nas relações sociais. Com exceção de um dos participantes do presente estudo, todos os demais não exerciam mais nenhuma atividade profissional. Sendo assim, considera-se que a não manutenção da vida laboral, pela condição física, também pode ser causadora de isolamento social, devido ao papel exercido pelo desempenho ocupacional (Teixeira et al., 2017).

Tendo em vista o processo crescente de envelhecimento da população mundial, torna-se necessário aprofundar a reflexão acerca das repercussões do suporte social para portadores de insuficiência cardíaca. A partir das considerações apresentadas neste trabalho, espera-se contribuir para implementar estratégias e ações que incluam os aspectos psicológicos e emocionais do adoecimento. 


\section{CONSIDERAÇÕES FINAIS}

O presente estudo contribuiu para aprofundar a compreensão da visão dos pacientes sobre a repercussão do suporte social recebido. Notou-se que o suporte social percebido como adequado pela maioria dos pacientes, mostrou ser um fator de grande importância para a saúde física e também emocional dos participantes.

Em relação às limitações do estudo, pode-se citar o pequeno número de pacientes, que não permite a generalização dos dados para a população portadora de IC. Por se tratar de estudo qualitativo buscou aprofundar a reflexão acerca das repercussões do adoecimento diante do envelhecimento e sua ligação com o suporte social percebido. Cabe ressaltar que os participantes integravam um programa multidisciplinar de reabilitação e faziam parte de grupos em que receberam intervenções de apoio e psicoeducação sobre a cardiopatia, o que pode ter influenciado em algum momento as respostas dos mesmos, embora o suporte social recebido por profissionais não tenha sido destacado.

Finalmente, espera-se que este trabalho possa contribuir como subsídio para futuras intervenções grupais ou individuais com pacientes portadores da IC, já que traça algumas das necessidades em termos de suporte social, por parte dos pacientes idosos e pontos que necessitam de atenção. Futuros estudos podem investigar o suporte social de tais pacientes em populações maiores, bem como relatar a estrutura de intervenções interdisciplinares promotoras de saúde para essa população.

\section{REFERÊNCIAS}

Barbosa, R. R., Franklin, R. V., Stefenoni, A. V., Moraes, V. D. Jacques, T. M., Serpa, R. G., Calil, O. A., \& Barbosa, L. F. M. (2014). Análise da Qualidade de Vida em Homens e Mulheres Portadores de Insuficiência Cardíaca. Revista Brasileira de Cardiologia, 4(27), 97-103. Recuperado de: http://www.onlineijcs.org/sumario/27/pdf/v27n2a05.pdf

Bardin, L. (2016). Análise de conteúdo. (3ª Reimpressão). São Paulo, SP: Edições 70.

Bocchi, E. A., Marcondes-Braga F. G., Bacal, F., Ferraz, A. S., \& Albuquerque D., Rodrigues, D. A. (2012). Sociedade Brasileira de Cardiologia. Atualização da Diretriz Brasileira de Insuficiência Cardíaca Crônica. Arquivos Brasileiros de Cardiologia, 98(1), 1-33. Recuperado de: http://publicacoes.cardiol.br/consenso/2012/Diretriz\%20IC\%20Cr\%C3\%B4nica.pdf

Campos, E. P., Martins, W. A., \& Carvalho, R. C. W. C. (2019). Conhecendo melhor os cuidadores de pacientes com insuficiência cardíaca. Revista da Jopic, 02(04), 57-64. http://dx.doi.org/10.1590/S0066-782X2012000700001

Cestari, R.. F., Florêncio, R. S., Pessoa, V. L. M. de P., \& Moreira, T. M. M. (2021). Validação dos marcadores da vulnerabilidade em saúde da pessoa com insuficiência cardíaca. Revista Eletrônica Acervo Saúde, 13 (5). https://doi.org/10.25248/reas.e7282.2021

Dantas, R. A. S., Pelegrino, V. M., \& Garbin, L. M. (2007). Avaliação do apoio social e sua relação com variáveis sociodemográficas de pacientes com Insuficiência Cardíaca em seguimento ambulatorial. Ciência, Cuidado e Saúde, 6(4), 465-462. https://doi.org/10.4025/cienccuidsaude.v6i4.3680

Engel, C. L. (Coord.). (2011). Cardiologia. (vol. 2). Porto Alegre, RS: Medyklin Editora LTDA.

Fernandes, A. D. F, Fernandes, G. C., Mazza, M. R, Knijnik, L. M, Fernandes, G., \& Vilela, A. T., et al. (2020). Insuficiência cardíaca no Brasil subdesenvolvido: análise de tendência de dez anos. Arquivos Brasileiros de Cardiologia, 14(2), 222-31. https://doi.org/10.36660/abc.20180321 
Finkler, A. L., \& Vivian, A. G. (2018). Grupo focal de psicologia em pacientes com insuficiência cardíaca. Aletheia, 51(1-2), 80-96. https://doi.org/10.29327/226091

Hwang B ${ }^{\prime}$ Fleischmann K. E., Howie-Esquivel J , Stotts N. A with heart failure: impact on patients' families. Am J Crit Care, 20(6), 431-41. https://doi.org/10.4037/ajcc2011472

Ishigaki, B. S. V., Hanna, M. B. S., Castro, L. M., Holanda, V. B. T., \& Castro, R. C. (2021). Perfil eletrocardiográfico de pacientes com insuficiência cardíaca em um centro de referência. Pará Research Medical Journal, 5(3), 1-8. http://dx.doi.org/10.4322/prmj.2021.003

Marques, C. A., Stefanello, B., Mendonça, C. N., \& Furlanetto, L. M. (2013). Associação entre depressão, níveis de dor e falta de apoio social em pacientes internados em enfermarias de clínica médica. Jornal Brasileiro de Psiquiatria, 62(1), 1-7. https://doi.org/10.1590/S0047$\underline{20852013000100001}$

Mendes, A. M. O. C., \& Eufrásio, M. L. P. (2013). Análise compreensiva de uma intervenção na ansiedade e depressão em doentes hospitalizados com insuficiência cardíaca. Revista de Enfermagem Referência, 11, 29-35. http://dx.doi.org/10.12707/RIII1305

Mendes, A. P., Bastos, F., \& Paiva, A. (2010). A pessoa com Insuficiência Cardíaca. Factores que facilitam/dificultam a transição saúde/doença. Revista de Enfermagem Referência, 3(2), 7-16. https://dx.doi.org/10.12707/rii1049

Ministério da Saúde. (2019). Datasus: mortalidade por cardiopatias - 1996 a 2019, pela CID-10. Recuperado de: http://tabnet.datasus.gov.br/cgi/tabcgi.exe?sim/cnv/obt10uf.def

Molano-Barrera, D., \& González-Consuegra, R. (2021). Factores deteriorantes de la calidad de vida en insuficiencia cardiaca: revisión integrativa. Duazary, 18(1), 86-98. https://doi.org/10.21676/2389783X.3889

Mondesir, F. L., Carson, A. P., Durant, R. W., Lewis, M. W., Safford, M. M., \& Levitan, E. B. (2018). Association of functional and structural social support with medication adherence among individuals treated for coronary heart disease risk factors: Findings from the Reasons for Geographic and Racial Differences in Stroke (REGARDS) study. Plos One, 1-13. https://doi.org/10.1371/journal.pone.0198578

Oliveira, L. G., Souto, T. A., \& Vitorio, A. M. F. (2017). O cuidador de paciente com insuficiência cardíaca: desafios no cuidar. Revista Científica de Enfermagem, 7(19), 3-14 https://doi.org/10.24276/rrecien2358-3088.2017.7.19.3-14

Ornelas, J. (1994). Suporte social: origens, conceitos e áreas de investigação. Análise Psicológica, 2-3 (7), 333-339. Recuperado de: http://repositorio.ispa.pt/bitstream/10400.12/3103/1/1994 23 333.pdf

Possatto, J. de M., \& Rabelo. D. F. (2017). Condições de saúde psicológica, capacidade funcional e suporte social de idosos. Revista Kairós, 20(2), 45-58. https://doi.org/10.23925/2176901X.2017v20i2p45-58

Queiroz, M. E. G., \& Foz, M. L. F. N. N. (2018). Atenção integrada à pessoa com insuficiência cardíaca na perspectiva terapêutico ocupacional e psicológica: um relato de experiência. REFACS, 6(1), 123-129. https://doi.org/10.18554/refacs.v6i1.2374

Queiroz, L. M. N. de, Viana, R. E. N, Araujo, S. A. de, Pedrosa, I. L., Araujo, A. A. de (2021). Perfil de idosos admitidos nas unidades hospitalares por insuficiência cardíaca que reinternam. Brazilian Journal of Develpment, 7(1), 6084-6094. https://doi.org/10.34117/bjd7n1-413

Rezende, L. K., Mendes, I. J. M., \& Santos, B. M. O. (2007). Suporte social para idosos portadores de insuficiência cardíaca. Revista de Ciências Farmacêuticas Básica e Aplicada, 28(1), 107-111.

Rodrigues, M. A., \& Seidl, E. M. F. (2008). A importância do apoio social em pacientes coronarianos. Padéia, 18(40), 279-288. https://doi.org/10.1590/S0103-863X2008000200006

Ribeiro, J. L. P. (1999). Escala de Satisfação com o Suporte Social (ESSS). Análise psicológica, 3(8), 547558. Recuperado de: http://www.scielo.mec.pt/pdf/aps/v17n3/v17n3a10.pdf

Silva, B. de S. M. M., Marinho, S. C., Silva, S. E.., Dos Santos Silva, R., Da Silva Pires, C. G.., \& Nascimento, E F. (2021). Qualidade de vida de idosos com insuficiência cardíaca. Ciencia y Enfermería, 27, 1-8. https://doi.org/10.9789/2175-5361.2010.v0i0.\%25p

Soares, D. A., Toledo, J. A. S., Santos, L. F., Lima, R. M. B., \& Galdeano, L. E. (2008). Qualidade de vida de portadores de insuficiência cardíaca. Acta Paulista de Enfermagem, 21(2), 243-248. https://doi.org/10.1590/S0103-21002008000200002

Santos, A. C. S., Santo, F. H. E., Pestana, L., Daher, D. V., \& Santana, R. (2011). Insuficiência cardíaca: estratégias usadas por idosos na busca por qualidade de vida. Revista Brasileira de Enfermagem, 64(5), 857-863. https://doi.org/10.1590/S0034-71672011000500009 
Teixeira, E. S., Masuchi, M. E., \& Correia, R. L. (2017). Desempenho dos papeis ocupacionais em cardiopatas em período de hospitalização e pós-hospitalização. Revista Interinstitucional Brasileira de Terapia Ocupacional, 1(3), 353-365. http://dx.doi.org/10.47222/25263544.rbto7920

Tinoco, J. de . M. V. P, Souza, B. P. e S. de, Oliveira, S. X. de, Oliveira, J. A. de, Mesquita, E. T., \& Cavalcanti, A. C. D. (2021). Associação entre sintomas depressivos e qualidade de vida em pacientes ambulatoriais e internados com insuficiência cardíaca. Revista Da Escola De Enfermagem Da USP, 55, (3686). https://doi.org/10.1590/S1980-220X2019030903686

\section{CONFLITOS DE INTERESSES}

Não há conflitos de interesses.

\section{SOBRE OS AUTORES}

Verônica Schneider Röder é psicóloga clínica graduada pela Universidade Luterana do Brasil.

E-mail: veronicaroder96@yahoo.com.br

(1) https://orcid.org/0000-0003-2770-9485

Aline Groff Vivian é psicóloga clínica, Mestre e Doutora em Psicologia pela Universidade Federal do Rio Grande do Sul. Professora do Curso de Psicologia e do Programa de Pós-Graduação em Promoção da Saúde da Universidade Luterana do Brasil, Canoas/RS.

E-mail: alinegvivian@gmail.com

(1) https://orcid.org/0000-0003-2628-629x 